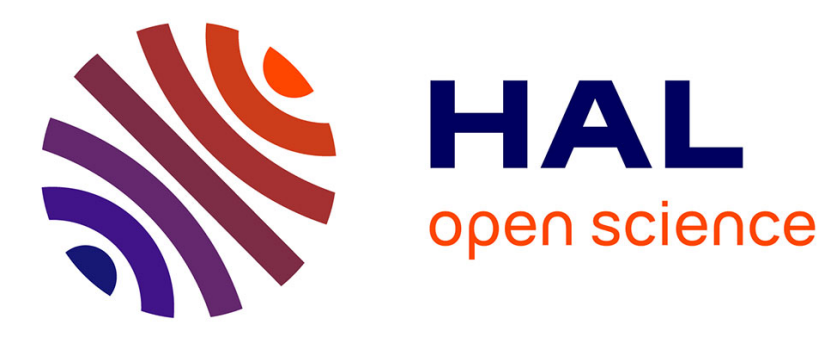

\title{
Remote Collaboration across Heterogeneous Large Interactive Spaces
}

\author{
Cédric Fleury, Nicolas Ferey, Jean-Marc Vézien, Patrick Bourdot
}

\section{To cite this version:}

Cédric Fleury, Nicolas Ferey, Jean-Marc Vézien, Patrick Bourdot. Remote Collaboration across Heterogeneous Large Interactive Spaces. IEEE VR International Workshop on 3D Collaborative Virtual Environments (3DCVE 2015), Mar 2015, Arles, France. 10.1109/3DCVE.2015.7153591 . hal01242306

\section{HAL Id: hal-01242306 \\ https://hal.inria.fr/hal-01242306}

Submitted on 11 Dec 2015

HAL is a multi-disciplinary open access archive for the deposit and dissemination of scientific research documents, whether they are published or not. The documents may come from teaching and research institutions in France or abroad, or from public or private research centers.
L'archive ouverte pluridisciplinaire HAL, est destinée au dépôt et à la diffusion de documents scientifiques de niveau recherche, publiés ou non, émanant des établissements d'enseignement et de recherche français ou étrangers, des laboratoires publics ou privés. 


\title{
Remote Collaboration across Heterogeneous Large Interactive Spaces
}

\author{
Cédric Fleury ${ }^{1} \quad$ Nicolas Férey ${ }^{2}$ Jean-Marc Vézien ${ }^{2}$ \\ ${ }^{1}$ Université Paris-Sud \& CNRS (LRI), Inria, Orsay (France) \\ 2 VENISE group, CNRS-LIMSI, Orsay (France) *
}

Patrick Bourdot ${ }^{2}$

\begin{abstract}
Immersive virtual reality systems or high resolution wall-sized displays become more common for analyzing the increasing among of data from science, industry, business and society. These large interactive spaces are powerful tools to enable remote users to work together on shared data. However, we cannot imagine that remote collaboration in such systems becomes wide-spread if it requires that all users have the exact same physical devices, and we need to make remote collaboration across different systems possible. The asymmetric interaction capabilities of each user are also an interesting opportunity to develop new collaboration strategies. In this short position paper, we present our past work about collaborative virtual environments and, in particular, how to represent the physical environments of each user in a virtual environment. We also introduce an on-going project which aims to support remote collaborative interaction across heterogeneous large interactive spaces.
\end{abstract}

Index Terms: I.3.7 [Computer Graphics]: 3D Graphics and Realism-Virtual Reality; H.5.3 [Information Interfaces and Presentation]: Group and Organization Interfaces-CSCW

\section{INTRODUCTION}

More and more large datasets including scientific data or CAD models (train, aircraft, etc.) have to be visualized by remote experts. These datasets are complex to visualize and manage. Consequently, large interactive spaces with specific visualization systems such as high resolution wall-sized displays or immersive virtual reality systems are becoming more common to deal with large datasets. The possibility to display large amounts of information, potentially in $3 \mathrm{D}$, and to improve the spatial organization of this information offers new opportunities to manage the complexity of these datasets.

Remote collaboration across interconnected large interactive spaces enables remote experts to deal with the complexity of the tasks, combine expertise, share knowledge, but also take advantage of the different features of their visualization systems. For example, some experts can have a 3D view of the data using an immersive virtual reality system, while some others can have a wider view of the data or a view of the data at different times using a high resolution wall-sized display. Even if remote users do not have the same interaction capabilities (2D, 3D, tactile, etc.), they still need to be able to interact together and to understand what the others are doing. The main challenge of remote collaboration across large interactive spaces is to enable all the users to interact together by leveraging the particular interaction capabilities of each physical system. Designing interaction for such asymmetric capabilities will lead to new collaboration strategies for large interactive spaces.

\section{Previous work}

In previous work, we have studied remote collaboration in virtual environments, covering both collaborative interaction among remote users and technical aspects of distributed virtual environments including data distribution [6] and software architecture [3].

*e-mails: cfleury@ $\overline{1 r i . f r,\{f e r e y, ~ v e z i e n, ~ b o u r d o t\} @ 1 i m s i . f r ~}$

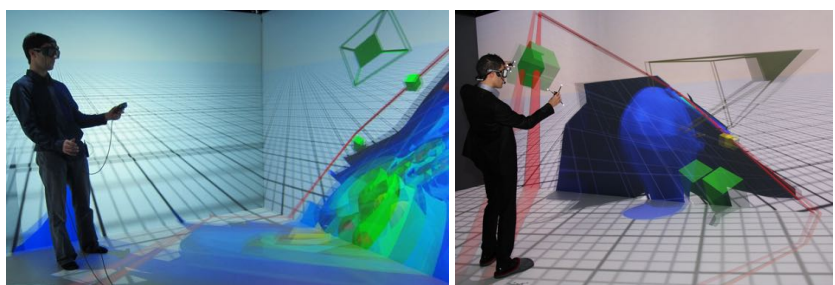

Figure 1: Remote collaboration across two immersive virtual reality systems located in Rennes and London (scientific data analysis) [7].

We ran experiments to study collaborative manipulation techniques for analyzing scientific data among users located in two interconnected immersive virtual reality systems in Rennes (France) and London [7] (Figure 1). These experiments aimed to compare a remote collaborative manipulation technique with a single user manipulation technique for positioning a clipping plane to perform a precise cross-section of scientific data. The experiments has shown that the remote collaborative manipulation technique was significantly more efficient than the single user manipulation when the manipulation task was more difficult and required more precision.

We have also investigated the feeling of presence in virtual environment and the awareness of the other users in collaborative virtual environment. We think that it is important for each user to understand what his/her own interaction capabilities are, but also what the interaction capabilities of the other users are in the virtual environment. In [5], we proposed the Immersive Interactive Virtual Cabin (IIVC) model to describe these interaction capabilities according the hardware devices used by each user, and to obtain a virtual representation of the users' physical environments. This 3D abstract representation of physical features is useful to improve the mutual understanding between users. In [4], we illustrated how the IIVC model can be used in a collaborative navigation task, and we presented how we can add 3D representations of 2D interaction tools to deal with asymmetrical collaborative configurations, providing $3 \mathrm{D}$ cues for a user to understand the actions of others even if he/she is not fully immersed in the shared CVE.

Finally, we have studied co-located collaboration in multistereoscopic immersive virtual reality systems and, in particular, how to manage perceptual conflicts such as occlusion [1] or cohabitation for individual navigation tasks [2] when users share the same interaction spaces. Since each user has his own particular view of the virtual environment in such systems because of the multistereoscopic display, this collaboration mode is widely related to remote collaboration. It would be interesting to investigate these two collaboration modes in parallel and to compare them in the context of different large interactive spaces.

\section{Collaboration across heterogeneous systems}

We have started a large project, called DIGISCOPE, to create a high-performance visualization infrastructure for collaborative interaction with extremely large datasets and computations in the context of scientific data analyses, design, engineering, decisionsupport, education and training. DIGISCOPE consists of ten interconnected large interactive spaces, including virtual reality systems, 3D display devices, large wall-sized displays and a variety of interaction devices such as motion trackers. One of the main goals of DIGISCOPE is to create a unique infrastructure to develop and 


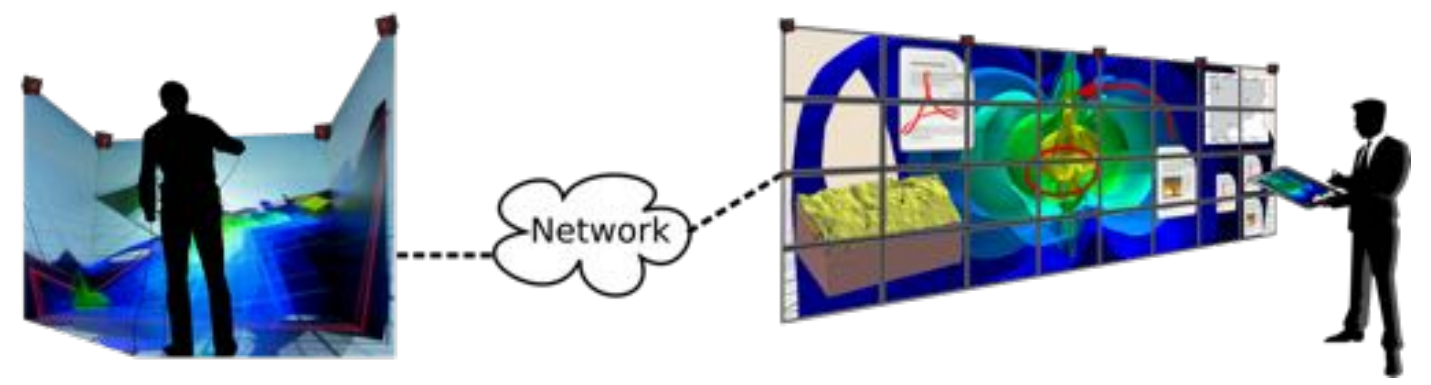

Figure 2: Users in a 3D virtual reality system (left) and in front of a 2D wall-sized display (right) are analyzing together scientific data.

study remote collaboration systems for interconnected large interactive spaces. At the time of this writing, nine of the ten rooms are operational and the system interconnecting them is being created.

In the context of DIGISCOPE, we want to design interfaces that consider collaboration as a fundamental feature of the system: users should be able to act together on shared contents, but also to understand what the others are seeing or doing, and what their interaction capabilities are. Understanding the current activity of other users is crucial for remote collaboration across heterogeneous large interactive spaces. We will investigate novel collaborative interfaces that enable users both to perform collaborative manipulation, but also to communicate with each other to show something to the others, share a particular viewpoint, annotate shared data, etc.

Users in different interactive spaces usually do not have the same interaction capabilities depending of the configuration of their physical devices. For instance, users in front of a wall-sized display interact using 2D devices, while users in an immersive virtual reality system use 3D interaction techniques. An efficient remote collaboration is possible in this case only if users can interact with the shared contents with both 2D and 3D techniques. Consequently, collaborative interfaces should deal with a wide variety of physical devices and use cases. We aim to develop technical tools to design interaction techniques suitable with multiple physical environments and user requirements. For example, we are currently investigating techniques for pointing on targets in dense environment such as molecular simulation, that are suitable with both immersive virtual reality systems and wall-sized displays [8].

\section{2D / 3D Collaborative INTERACtion}

We have started to work on this project by deploying a 3D virtual environment on two different large interactive spaces (Figure 2):

- The EVE platform is an immersive virtual reality system (CAVE) composed of four back-projected stereoscopic screens: $4.8 \times 2.7 m$ (front $\&$ flour), $2.7 \times 2.7 m$ (left $\&$ right). A 3D tracking system is used to track the user head and hands.

- The WILD platform is a high resolution 2D wall-sized display $(5.5 \times 1.8 \mathrm{~m}, 20480 \times 6400$ pixels $)$ made of a grid of $8 \times 430$ " monitors. A 3D tracking system and 2D tactile devices can be used for interaction.

The virtual environment is similar to the one used for the experiments about scientific data analysis in [7] (section 2), and is based on the data distribution proposed in [6] and the software architecture proposed in [3]. In the original experiments, a collaborative 3D manipulation task was performed by two remote users in two immersive virtual reality systems, but the users have now to perform the same task even if one is in front of a 2D wall-sized display.

This asymmetric collaboration arises several questions: how can we design interaction techniques to enable users to perform the same task with both 2D and 3D devices, free gestures and haptic feedback, 2D and stereoscopic vision? Can we find a way to make this design process more generic and independent from the task and from the physical devices? How can the users be as efficient with these different interaction techniques? Even if their interaction techniques are not the same according to their physical devices, we want that all users can be equally involved in the task. We should also consider how to represent users in front of a wallsized display or in an immersive virtual reality system to give to the others a sense of both what they are doing and what they can do.

\section{Conclusion}

To make remote collaboration across large interactive spaces possible, remote users need to interact together even if they do not use the same physical systems. They must be able both to act on shared contents, but also to understand what are the activity and the capabilities of the other users. The DIGISCOPE project is an unique opportunity to explore remote collaboration across ten interconnected heterogeneous platforms. In particular, we have started to study how users in a immersive virtual reality system can collaborate with users in front of a 2D wall-sized display on a shared task. We also want investigate how these asymmetric interaction systems can lead to new collaboration strategies.

\section{ACKNOWLEDGEMENTS}

Part of this work is supported by the French National Research Agency grant ANR-10-EQPX-26-01 "DIGISCOPE".

\section{REFERENCES}

[1] W. Chen, N. C. Clavel, Férey, and P. Bourdot. Perceptual conflicts in a multi-stereoscopic immersive virtual environment: Case study on face-to-face interaction through an avatar. Presence: Teleoperators and Virtual Environments, 23(4), Dec 2014, in press.

[2] W. Chen, N. Ladévèze, C. Clavel, D. Mestre, and P. Bourdot. User cohabitation in multi-stereoscopic immersive virtual environment for individual navigation tasks. In Proc. of the Virtual Reality conference (IEEE VR). IEEE, 2015, to appear.

[3] T. Duval and C. Fleury. PAC-C3D: A new software architectural model for designing 3d collaborative virtual environments. In Proc. of Int. Conference on Artificial Reality and Telexistence (ICAT), 2011.

[4] T. Duval, T. T. H. Nguyen, C. Fleury, A. Chauffaut, G. Dumont, and V. Gouranton. Improving awareness for $3 \mathrm{~d}$ virtual collaboration by embedding the features of users' physical environments and by augmenting interaction tools with cognitive feedback cues. Journal on Multimodal User Interfaces, 8(2):187-197, June 2014.

[5] C. Fleury, A. Chauffaut, T. Duval, V. Gouranton, and B. Arnaldi. A generic model for embedding users physical workspaces into multiscale collaborative virtual environments. In Proc. of Int. Conference on Artificial Reality and Telexistence (ICAT), 2010.

[6] C. Fleury, T. Duval, V. Gouranton, and B. Arnaldi. A new adaptive data distribution model for consistency maintenance in collaborative virtual environments. In Proc. of Joint Virtual Reality Conference (JVRC), pages 29-36. Eurographics Association, 2010.

[7] C. Fleury, T. Duval, V. Gouranton, and A. Steed. Evaluation of remote collaborative manipulation for scientific data analysis. In Proc. of Symposium on Virtual Reality Software and Technology (VRST), pages 129-136. ACM, 2012.

[8] A. Kouyoumdjian, P. Bourdot, S. Huot, and N. Férey. Understanding picking of moving targets: towards new paradigms for interactive and immersive molecular simulations. Faraday Discussion 169, 2014. 